\title{
The Algebraic and Ordering Construction of Lattice Hongying $\mathrm{Mi}^{1,}$, , Kunlong Zhang ${ }^{1, \mathrm{~b}}$ \\ ${ }^{1}$ Faculty of Science, Minzu University of China, Beijing, 100081, China \\ aemail:mihongying@aliyun.com, bemail:zhangkunl@126.com
}

Keywords: Ordering; Map; Lattice; Sublattice

\begin{abstract}
Based on the order theory, a study of the differences between the preordering and the ordering and a discussion of the isotone map and homomorphism map are obtained. According to the differences of the constructions of the algebraic lattice and the ordering lattice, we prove the algebraic sublattice and the ordering sublattice are equivalent with the strengthen condition.
\end{abstract}

\section{Introduction}

George Grätzer gives a concise development of the basic concepts of lattice theory. Generally, our focus is on lattice structure theory, however, this paper intends to illustrate if the algebraic sublattice and the ordering sublattice are equivalent. We draw conclusions from "General Lattice Theory”, “The Congruence of a Finite Lattice”[3] by George Grätzer:

\section{Theorem 1}

(1)Let the poset $L=(L, \leq)$ be a lattice. Set $a \wedge b=\inf \{a, b\}, a \vee b=\sup \{a, b\}$.Then the algebra $L^{a}=(L, \wedge, \vee)$ is a lattice.

(2)Let the algebra $L=(L, \wedge, \vee)$ be a lattice. Set $a \leq b$ iff $a \wedge b=a$.Then $L^{P}=(L, \leq)$ is a poset, and the poset is a lattice.

(3)Let the poset $L=(L, \leq)$ be a lattice. Then $\left(L^{a}\right)^{P}=L$.

(4)Let the algebra $L=(L, \wedge, \vee)$ be a lattice. Then $\left(L^{P}\right)^{a}=L$.

In other words, a lattice as an algebra and a lattice as a poset are “equivalent” concepts. We wonder if the concepts of a sublattice as an algebra and a sublattice as a poset are the same. We will discuss about it later.

Theorem 2 [1]Every homomorphism map is an isotone map.

Theorem 3 Let $P$ be a finite order. Then $a \leq b$ if and only if $a=b$ or if there exists a finite sequence of elements $x_{1}, x_{2}, \cdots, x_{n}$ such that $a=x_{1} \prec x_{2} \prec \cdots \prec x_{n}=b$.

\section{Preliminaries}

Definition 1 [2] A preorder is a nonempty set $Q$ with a binary relation $\leq$ that is reflexive and transitive.

Definition 2 A partially ordered set is a system consisting of a nonempty set $P$ and a binary relation $\leq$ in $P$ such that the following conditions are satisfied for all $x, y, z \in P$ :

(1) Reflexivity $x \leq x$

(2) Antisymmetry $x \leq y, y \leq x \Rightarrow x=y$

(3) Transitivity $x \leq y, y \leq z \Rightarrow x \leq z$

The relation $\leq$ is a partial order in the set $P$, and $P$ is said to be partially ordered by the relation $\leq$.

Definition $3(P, \leq)$ is a poset, $S$ is a subset of $P, a \in P$ :

(1)If $\forall s \in S, a \leq s(s \leq a)$, then $a$ is a lower(upper) bound of $S$;

(2)If $\forall s \in S, a \in S, a \leq s(s \leq a)$, then $a$ is called the least(greatest) element of $S$; 
(3)A lower bound $a$ of $S$ is the greatest lower bounder of $S$, for any lower bound $b$ of $S$,we have $a \geq b$. An upper bound $a$ of $S$ is the least upper bounder of $S$, for any upper bound $b$ of $S$, we have $a \leq b$.

Definition 4 [5] A poset $L=(L, \leq)$ is a lattice, if $a, b \in L, \sup \{a, b\}$ and $\inf \{a, b\}$ exist.

Definition 5 If $L$ is a lattice, then it is readily verified that the following conditions hold for all $a, b, c \in L$
(1) $a \wedge a=a, a \vee a=a$
(2) $a \wedge b=b \wedge a, a \vee b=b \vee a$
(3) $(a \wedge b) \vee a=a,(a \vee b) \wedge a=a$
(4) $(a \wedge b) \wedge c=a \wedge(b \wedge c),(a \vee b) \vee c=a \vee(b \vee c)$

Definition 6 A lattice $L_{1}=\left(L_{1}, \wedge, \vee\right)$ is called a algebraic sublattice of $L=(L, \wedge, \vee)$ if it satisfies the following two conditions:

(1) $L_{1} \subseteq L$ is a nonvoid subset of $L$

(2) $L_{1}$ is closed under the same $\vee$ and $\wedge$

Definition 7 A lattice $L_{1}=\left(L_{1}, \leq\right)$ is called a partially sublattice of $L=(L, \leq)$, if it satifies the following two conditions:

(1) $L_{1} \subseteq L$ is a nonvoid subset of $L$

(2) $\left(L_{1}, \leq\right)$ is a suborder of $(L, \leq)$

Definition 8 A homomorphism $\varphi$ of the lattice $L_{0}$ into a lattice $L_{1}$ is a map of $L_{0}$ into $L_{1}$ satisfying both $\varphi(a \vee b)=\varphi(a) \vee \varphi(b)$ and $\varphi(a \wedge b)=\varphi(a) \wedge \varphi(b)$.

Definition 9 The map $\varphi: P_{0} \rightarrow P_{1}$ is an isotone map of the poset $P_{0}$ into the poset $P_{1}$ iff $a \leq b$ in $P_{0}$ implies that $\varphi(a) \leq \varphi(b)$ in $P_{1}$.

Definition 10 A chain is an order with no incomparable elemets.

Definition 11 There exists a finite sequence of elements $x_{1}, \cdots, x_{n} \in Q$ such that $x_{1} \prec \cdots \prec x_{n} \prec x_{1}(\mathrm{n}>1)$, then $Q$ is called a circle.

Definition 12 The subset $K$ of the lattice $L$ is called convex iff $a, b \in K, c \in L$, and $a \leq c \leq b$ imply that $c \in K$.

\section{Algebraic Sublattices and Partial Sublattices}

As we know, a lattice as an algebra and a lattice as a poset are "equivalent" concepts. There are some differences between algebraic construction and partial construction [4] .

Theorem $1 L_{1}$ is a partial sublattice of $L$,if $\forall a, b \in L_{1}, \inf \{a, b\}, \sup \{a, b\} \in L_{1}$ and $\inf \{a, b\}$, $\sup \{a, b\} \in L$ are equivalent, then partial sublattice $L_{1}$ and algebraic sublattice $L_{1}$ are equivalent. Proof: Let $L$ be a lattice, $L_{1}$ is an algebraic sublattice of $L . L_{1}$ is also a lattice. The conclusion of theorem1, $L_{1}^{P}$ is a partial lattice. In view of the results, $a \leq b \Leftrightarrow a \wedge b=a$. Certainly $(a \wedge b) \wedge a=a \wedge b$ implies $a \wedge b \leq a$. Similarly $\quad(a \wedge b) \wedge b=a \wedge b$ implies $a \wedge b \leq b$.It is very easy to infer $a \wedge b$ is a lower bound of $a, b$. Let $d$ be a lower bounder of $a, b$, so $d \leq a$ and $d \leq b$. Certainly $d \wedge a=d$ and $d \wedge b=d$, thus we can infer $d \wedge(a \wedge b)=(d \wedge a) \wedge b=d \wedge b=d$. So we get $d \leq a \wedge b$, then $a \wedge b$ is the greatest lower bound of $a, b$ in $L_{1}^{P}$.In the definition of algebraic sublattice, $a \wedge b$ is also the greatest lower bound of $a, b$ in $L$.

In view of the results, $a \leq b \Leftrightarrow a \wedge b=a \Leftrightarrow b=a \vee b$. Since $(a \vee b) \vee a=a \vee b$, we infer 
$a \leq a \vee b$. Obviously $(a \vee b) \vee b=a \vee b$ implies $b \leq a \vee b$.we can also infer that $a \vee b$ is an upper bound of $a, b$. Let $d^{\prime}$ is an upper bound of $a, b$.Since $a \leq d^{\prime}$ and $b \leq d^{\prime}$, thus $a \vee d^{\prime}=d^{\prime}$ and $b \vee d^{\prime}=d^{\prime}$, thus $d^{\prime} \vee(a \vee b)=\left(d^{\prime} \vee a\right) \vee b=d^{\prime} \vee b=d^{\prime}$. Thus $a \vee b$ is the least upper bound of $a, b$ in $L_{1}^{P}$ and in $L$.

We conclude that $L_{1}$ is a partial sublattice of $L$, if $\forall a, b \in L_{1}, \inf \{a, b\}, \sup \{a, b\} \in L_{1}$ and $\inf \{a, b\}, \sup \{a, b\} \in L$ are equivalent, then partial sublattice $L_{1}$ and algebraic sublattice $L_{1}$ are equivalent.

Theorem 2 Every algebraic sublattive is the partial sublattice.

Proof: Let $L$ be a lattice and $L_{1}$ be a partial sublattice of $L$.Assume $L_{1}$ does not satisfy the definition of partial sublattice. However, $\forall a, b \in L_{1}, a \wedge b, a \vee b \in L_{1}$, in the view of the preceding results, we get the conclusion that $a \wedge b=\inf \{a, b\}, a \vee b=\sup \{a, b\}$, contrary to the assumption. Consequently, every algebraic sublattice is the partial sublattice.

Theorem 3 Every convex partial sublattice is the partial sublattice.

Proof: Let $K$ be the convex partial sublattice of $L, a \leq b \in K$ for all $c \in[a, b]$ satisfies $c \in K$. Assume that $K$ does not satisfy the definition of algebraic sublattice. There exists $a, b \in K$ but $a \wedge b$ or $a \vee b \notin K$. Thus $a \leq b \in K, c_{1}, c_{2} \in[a, b]$, and $a \leq c_{1} \leq c_{2} \leq b$. In the view of the preceding results, $a \leq b \Leftrightarrow a \wedge b=a$ and $a \vee b=b$, so we infer that $c_{1} \wedge c_{2}=c_{1}$ and $c_{1} \vee c_{2}=c_{2} \in K$, contrary to the assumption . Therefore every convex partial sublattice is the partial sublattice.

In fact, we can have the more general conclusion:

Theorem 4 Let $L_{1}$ be a parital sublattice of $L=(L, \wedge, \vee)$, then $L_{1}$ is an algebraic sublattice iff for all $a, b \in L_{1}$ satisfy $a \wedge b$ and $a \vee b \in L_{1}(\wedge, \vee$ in $L)$.

Proof: $\Rightarrow$ obviously.

$\Leftarrow L_{1}$ is a partial sublattice, according to the definition, for all $a, b \in L_{1}$, then $\inf \{\mathrm{a}, \mathrm{b}\}$ and $\sup \{\mathrm{a}, \mathrm{b}\} \in L_{1}$. Let $d=\inf \{a, b\} \in L_{1}$, according to the theorem, $d^{\prime}=\inf \{a, b\}=a \wedge b \in L$. Since $d^{\prime} \in L_{1}$, thus $d \geq d^{\prime}$. If $d>d^{\prime}$, contrary to the fact $d=\inf \{a, b\} \in L_{1}$, thus $d=d^{\prime}$.

Similarly, for all $a, b \in L_{1}$, let $d=\sup \{a, b\} \in L_{1}$ and $d^{\prime}=\sup \{a, b\}=a \vee b \in L$. Since $d^{\prime} \in L_{1}$, so $d \leq d^{\prime}$. If $d<d^{\prime}$, contrary to the fact $d=\sup \{a, b\} \in L_{1}$, therefore $d=d^{\prime}$.

\section{Ordering and its Properties}

A preorder is a nonempty set $Q$ with a binary relation $\leq$ that is reflexive and transitive. We can add some conditions on the preorder in order that the preorder and the poset are equivalent.

Theorem 1 The finite preorder is a poset iff there does not exist circle.

Proof: Assume that there is a circle $a \prec x_{0} \prec \cdots \prec x_{n} \prec a$.We get $a \leq x_{0}$, according to the transitivity , $x_{0} \leq a$. Combining the antisymmetry, $a \leq b, b \leq a \Rightarrow a=b$, then $a=x_{0}$, contrary to $a \prec x_{0}$.

For all $a, b, a \leq b$ and $b \leq a$, we have two situations:

(1) $a=b$

(2) $a \neq b$, then $a \prec x_{1} \prec \cdots \prec b$ and $b \prec x_{1}^{\prime} \prec \cdots \prec a$.

If $a \neq b$, we get $a \prec x_{1} \prec \cdots \prec b \prec x_{1}^{\prime} \prec \cdots \prec a$,contrary to the fact that there does not exist circle.

Therefore, for all $a, b$ if $a \leq b$ and $b \leq a$, we have $a=b$. 
Theorem 2 Every isotone map of the finite chain is a homomorphism.

Proof: Let $C$ be a finite chain. There exists a finite sequence of elements $x_{1}, x_{2}, \cdots, x_{n} \in C$ such that $x_{1} \prec x_{2} \prec \cdots \prec x_{n}$. Since $\varphi$ is an isotone map of $C$ into $P$, thus $\varphi\left(x_{1}\right) \prec \varphi\left(x_{2}\right) \prec \cdots \prec \varphi\left(x_{n}\right)$. For all $x_{1}, x_{2} \in C$ if $x_{1} \prec x_{2}$, we infer that $x_{1} \wedge x_{2}=x_{1}$ and $x_{1} \vee x_{2}=x_{2}$. Similarly, for all $\varphi\left(x_{1}\right), \varphi\left(x_{2}\right) \in P$, we get $\varphi\left(x_{1}\right) \wedge \varphi\left(x_{2}\right)=\varphi\left(x_{1}\right)$ and $\varphi\left(x_{1}\right) \vee \varphi\left(x_{2}\right)=\varphi\left(x_{2}\right)$.

For all $a, b \in C$, there are only two situations, $a \prec b$ or $b \prec a$. If $a \prec b$, then $\varphi(a \wedge b)=\varphi(a)=\varphi(a) \wedge \varphi(b)$ and $\varphi(a \vee b)=\varphi(a)=\varphi(a) \vee \varphi(b)$.

On the other hand, if $b \prec a$, then $\varphi(b \wedge a)=\varphi(b)=\varphi(b) \wedge \varphi(a)$ and $\varphi(b \vee a)=\varphi(b)=\varphi(b) \vee \varphi(a)$. Therefore every isotone map of the finite chain is a homomorphism.

\section{References}

[1] Chenjie. Elementary of Lattice Theory [M],_Hohhot:_Inner Mongolia University Press, 1990.

[2] Fangjie, Lattice Theory Guide [M].Beijing: China Higher Education Press, 2014.

[3] Gratzer G. The Congruence of a Finite Lattice[M].Academic Press, New York, 2006.

[4] Peter Crawley. Algebraic Theory of Lattices [M]. The United States of America, 1973.

[5] G.Birkhoff. Lattice Theory[M].Academic Press, New York, San Francisco.1940. 